\title{
Theme: Pediatric Gastroenterology
}

Gelatin tannate in acute diarrhea (Med Sci Monit 2017, 23: 2029-34)

Search for a safe agent that could effectively control symptoms and curtail the duration of acute diarrhea in children has been continuing since time immemorial. In this double-blind randomized controlled study, the efficacy of gelatin tannate, a mucoprotective agent, was evaluated against placebo in children under 1 year of age presenting with acute diarrhea. It was observed that within 12 hours of the intervention, there was a significant improvement in consistency and reduction in stool frequency in the gelatin group as compared to the placebo group. If the results could be verified in larger study group, we might be inching closer to devising the perfect therapeutic regimen for children with acute diarrhea.

Do probiotic foods really offer any health benefits? (Nutrients. 2017. April 19 9(4): pii: E400. doi: 10.3390/ nu9040400).

In this Canadian study, food label information program was used to identify probiotic-containing products in the food supply, and a comprehensive data base search was undertaken to find positive health effects of these probiotic strains documented through randomized controlled trials in humans. It was established that the six identified strains were associated with many positive health effects such as decreased diarrhea and constipation, antioxidant status, glycemic control, H. pylori eradication and enhanced immunity. However, it was also observed that most of these studies were funded by the food industry and tested dosages that were up to twenty five times the dosage found in most food products. It therefore emerges that probiotic-containing food products could have positive health benefits, but only if they contain effective probiotics in sufficient dosages so as to match the benefits demonstrated in clinical trials.

HMGB1, a new biomarker of celiac disease in children (Nutrition. 2017; 37:18-21)

Despite the availability of specific serology and point-of-care tests, the symptoms fluctuation as well as the "open-window" existing amongst the late and silent forms, often delays the diagnosis of celiac disease (CD). High mobility group box 1 (HMGB1), an important chromatin protein, mediates inflammation and gastrointestinal barrier failure. In this study involving 49 children with $\mathrm{CD}$ and 44 healthy children, antitissue transglutaminase type 2 and antideaminated form of gliadin antibodies, serum HMGB1 levels, and duodenal mucosal histopathology were performed. Serum HMGB1 levels were significantly higher in those with $\mathrm{CD}$ than those in the healthy control group $(P<0.001)$. Significant differences in serum HMGB1 levels were detected in children with typical CD form compared to both children with atypical $\mathrm{CD}$ form $(P<0.001)$ and children with silent $\mathrm{CD}$ form $(P<0.001)$. By using the Marsh classification, significant differences were also found between subjects with grade $3 \mathrm{~B}-\mathrm{B} 1$ and $3 \mathrm{C}-\mathrm{B} 2$ and villous atrophy, respectively $(P<0.05)$. On the contrary, no significant differences were detected in serum HMGB1 levels in subgroups of children with grade $3 \mathrm{~A}$ compared to grade $3 \mathrm{~B}-\mathrm{B} 1$. It was thus postulated that the HMGB1 is upregulated at diagnosis in all CD children, especially in typical form, and reflects the histologic severity of disease.

\section{Does rotavirus vaccination increase the risk of celiac disease or type 1 diabetes in children? (Pediatr Infect Dis J. 2017. April 10. doi: 10.1097/INF.0000000000001600)}

Rotavirus infection has been suggested as a trigger of type 1 diabetes (T1D) and celiac disease (CD) related autoimmunity in some studies. In this population-based cohort study in Finland, rotavirus vaccination records were collected from healthcare databases during 2009-2011 and validated for a sample of 495 children. Incident diagnoses of CD and TID during 20092014 in the cohort were identified in the National Care register. The adjusted relative risks $(95 \% \mathrm{CI})$ were $0.91(0.69,1.20)$ for $\mathrm{T} 1 \mathrm{D}$ and $0 \cdot 87(0 \cdot 65,1 \cdot 17)$ for $\mathrm{CD}$ in vaccinated children compared to unvaccinated, suggesting that oral rotavirus vaccination did not alter the risk of CD or T1D during four to six years follow-up after vaccination. Therefore, it appears that oral rotavirus vaccination is safe in the individuals at risk of CD and T1D.

D Serum biomarkers as predictors of complicated appendicitis in children (Pediatr Surg Int. 2017 Apr 29. doi: 10.1007/s00383-017-4088-1)

Choosing conservative mode of management of acute uncomplicated appendicitis without appendicolith over appendectomy is now an established treatment option. In this study, the authors evaluated whether commonly used serum biomarkers on admission could distinguish between simple and complicated appendicitis. Admission white blood cell (WBC), neutrophil (NEU), and C-reactive protein (CRP) levels were analyzed by ROC curve, and Kruskal-Wallis and contingency tests. Patients were divided according to age and histology [normal appendix (NA), simple appendicitis (SA), complicated appendicitis (CA)]. Of 1197 children (NA=186, SA=685, $\mathrm{CA}=326), 7 \%$ were $<5$ years, $55 \% 5-12$, and $38 \% 13-17$. CA patients had higher CRP and WBC levels than NA and SA $(P<0.0001)$. NEU levels were lower in NA compared to SA or CA $(P<0.0001)$, but were similar between SA and CA $(\mathrm{P}=0.6)$. CA patients had higher CRP and WBC levels than SA patients in 5-12$(\mathrm{P}<0.0001)$ and $13-17$-year groups $(P=0.0075, P=0.005)$, but not in $<5$-year group. CRP levels $>40 \mathrm{mg} / \mathrm{L}$ were found in $58 \%$ $\mathrm{CA}$ and $37 \%$ SA $(P<0.0001)$, and WBC count $>15 \times 10^{9} / \mathrm{L}$ in $58 \% \mathrm{CA}$ and $43 \% \mathrm{SA}(P<0.0001)$. Authors concluded that simple markers like CRP and WBC count may help the clinician predict complicated appendicitis in children older than 5 years age and guide decision-making as to the therapeutic modality.

VYOM AGGARWAL drvyomaggarwal@gmail.com 